\title{
Photonic streaking of attosecond pulse trains
}

\author{
Kyung Taec Kim, Chunmei Zhang', Thierry Ruchon², Jean-François Hergott' ${ }^{2}$, Thierry Auguste², \\ D. M. Villeneuve', P. B. Corkum ${ }^{1}$ and F. Quéré2^
}

\begin{abstract}
High harmonic radiation, produced when intense laser pulses interact with matter, is composed of a train of attosecond pulses. Individual pulses in this train carry information on ultrafast dynamics that vary from one half-optical-cycle to the next. Here, we demonstrate an all-optical photonic streaking measurement that provides direct experimental access to each attosecond pulse by mapping emission time onto propagation angle. This is achieved by inducing an ultrafast rotation of the instantaneous laser wavefront at the focus. We thus time-resolve attosecond pulse train generation, and hence the dynamics in the nonlinear medium itself. We apply photonic streaking to harmonic generation in gases and directly observe, for the first time, the influence of non-adiabatic electron dynamics and plasma formation on the generated attosecond pulse train. These experimental and numerical results also provide the first evidence of the generation of attosecond lighthouses in gases, which constitute ideal sources for attosecond pump-probe spectroscopy.
\end{abstract}

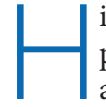
igh harmonic generation (HHG) in gases is a three-step process ${ }^{1}$, in which an electron wave packet is extracted from an atom or a molecule in each laser half-cycle, is accelerated by the laser field, and then recollides with its parent ion, thereby acting as an attosecond probe. Recently, HHG in gases has been used as a nonlinear spectroscopic technique to study the structure of molecular orbitals ${ }^{2}$, as well as orbital dynamics in excited systems ${ }^{3}$. For typical laser pulses, the measured harmonic spectrum is an average over multiple laser half-cycles ${ }^{4,5}$. Consequently, not only does the driving field vary during the pulse, but the probed medium can also evolve ${ }^{6,7}$. By dissecting the attosecond pulse train, we would achieve the temporal resolution needed to study the evolving nonlinear medium and the process of high-order harmonic generation itself.

A method to separate the attosecond pulses of a train in angle has been demonstrated numerically ${ }^{8}$ and experimentally ${ }^{9}$ for harmonics created from plasma mirrors. The basic principle relies on a temporal rotation of the laser-field instantaneous wavefront ${ }^{10}$ in the generation medium. Such a laser field produces a train where successive attosecond pulses are launched in different directions, and thus form spatially separated beams as they propagate away from the source. This so-called attosecond lighthouse delivers multiple synchronized beams consisting of isolated attosecond pulses ${ }^{9}$, and constitutes an ideal source of spatially separated, yet mutually coherent pulses for pump-probe spectroscopy with subfemtosecond resolution $^{11}$. Here, we demonstrate, numerically and experimentally, that attosecond lighthouses can also be obtained from HHG in gases. Then, because the lighthouse allows any attosecond pulse in the train to be accessed, we use it as a new ultrafast temporal measurement scheme-photonic streaking. We validate photonic streaking by directly measuring several essential temporal features of HHG in gases that have only previously been observed through much more involved diagnostics or analysis ${ }^{12,13}$, and then exploit this new, direct and simple technique for some new observations of the dynamics of $\mathrm{HHG}$ in gases.

\section{Wavefront rotation applied to HHG in gases}

Laser wavefront rotation (WFR) can be achieved by inducing a linear spatial chirp of the beam at its focus ${ }^{8,10}$, which in turn requires applying a spectral angular dispersion on the beam before focusing. In our experiment, where few-cycle phase-stabilized femtosecond laser pulses are used (see Methods for experimental details), this angular dispersion can be continuously controlled by rotating one wedge of the pair used to optimize pulse compression, as shown in Fig. 1a. The resulting spatially resolved spectrum of the laser pulse measured at the focus is shown in Fig. 1b. The central wavelength $\lambda(y)$ of the pulse varies across the focal spot, from $680 \mathrm{~nm}$ to $850 \mathrm{~nm}$ over the full-width at half-maximum (FWHM) of $42 \mu \mathrm{m}$, where $y$ is the direction transverse to the propagation direction $z$, as shown in Fig. 1a.

To illustrate the consequences of WFR on harmonic generation in gases, Fig. 1c-e presents model results (see Supplementary Section SI for details). The wavefront rotation of the laser field is visualized by following the zero crossings across the focal spot (shown as grey dashed lines in Fig. 1c). Due to this rotation, the wavefront of the attosecond pulses generated by this laser field also rotates from one half-cycle to the next, leading to different propagation directions for successive pulses, as illustrated by black arrows in Fig. 1c. An attosecond pulse generated at an earlier time propagates towards the top of the figure in Fig. 1d. The angular separation $\delta_{1 / 2}$ between two consecutive attosecond pulses is directly related to the central wavelength distribution $\lambda(y)$ by $\delta_{1 / 2}=\mathrm{d} \lambda(y) /(2 \mathrm{~d} y)$. From Fig. $1 \mathrm{~b}$ we predict $\delta_{1 / 2} \approx 2 \mathrm{mrad}$. When the divergence of each attosecond pulse is smaller than $\delta_{1 / 2}$, attosecond pulses generated during each half-cycle are spatially separated in the far-field, forming isolated attosecond pulses as shown in Fig. 1d. A clear signature of this effect is the observation of a continuous spectrum for each of these multiple beamlets, as simulated in Fig. 1e. In this simulation, the attosecond pulses originate from the short trajectory contribution to the dipole ${ }^{14}$. The long trajectory contribution is too weak to be seen due to its poor phase matching (see Supplementary Section SI-A for details).

For an experimental demonstration, we first use Ne as the nonlinear medium. For reference, the angularly resolved harmonic spectrum without any spatial chirp of the laser beam is shown in Fig. 2a. It is a typical harmonic spectrum with peaks regularly spaced by twice the laser photon energy, collimated in a single beam with a divergence of $2.2 \mathrm{mrad}$ (FWHM). As we rotate one wedge from

'Joint Attosecond Science Laboratory, National Research Council and University of Ottawa, 100 Sussex Drive, Ottawa ON K1A OR6, Canada, ${ }^{2}$ CEA, IRAMIS, Service des Photons Atomes et Molécules, F-91191 Gif-sur-Yvette, France. *e-mail: fabien.quere@cea.fr; kyungtaec.kim@nrc-cnrc.gc.ca 
a Gas jet
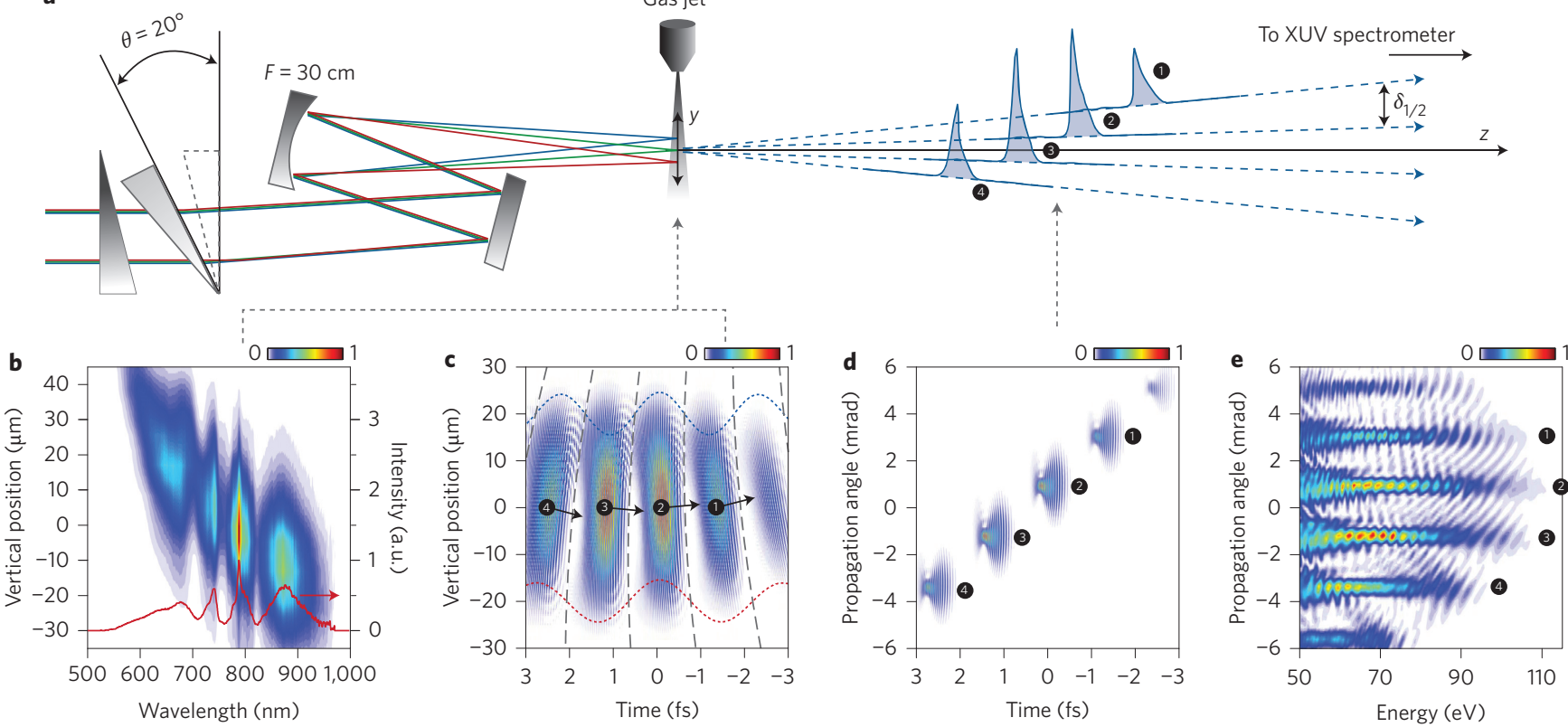

Figure 1 | Principle of attosecond lighthouses and photonic streaking in gases. a, Schematic of the experimental set-up. Angular dispersion is imposed on the laser beam before focusing using a misaligned pair of wedges, leading to spatial chirp at the focus. The attosecond pulses generated in each half-cycle of the laser pulse propagate in different directions. b. The spatially chirped laser spectrum (colour coded) measured as a function of the vertical position at the laser focus. The red line corresponds to the laser spectrum measured without spatial chirp. c-e, XUV radiation calculated with a laser beam that has the same amount of spatial chirp as measured in $\mathbf{b}$. The attosecond pulses generated in each half-optical-cycle are labelled by numbers to compare with other panels. In c, the temporal profile of the XUV radiation at the exit of the medium, $\left|E_{X}^{\text {near }}(t, y)\right|^{2}$, is shown, colour coded, as a function of time $t$ and vertical position $y$. The laser electric field at $y= \pm 20 \mu \mathrm{m}$ is shown by blue and red dotted lines. The zero crossing times of this field are shown by grey dashed lines. In $\mathbf{d}$, the temporal profile of the XUV radiation in the far-field, $\left|E_{X}^{\mathrm{far}}(t, \Theta)\right|^{2}$, is shown, colour coded, as a function of propagation angle $\Theta$. The intensity lineouts at the peak of each attosecond pulse are shown on top of the dashed arrows in $\mathbf{a}$. In $\mathbf{e}$, the angularly resolved XUV spectra of the multiple XUV beamlets in the far-field are shown.

$\theta=0^{\circ}$ to $20^{\circ}$, we observe that the beam splits into multiple spatially separated XUV beamlets (Fig. 2b). Four beamlets are detected, separated by $\sim 2.2 \mathrm{mrad}$, each weakly modulated spectrally. The $\sim 15 \%$ modulation amplitude is consistent with a slight spatial overlap of adjacent beamlets, which adds satellite pulses of $\sim 1 \%$ relative intensity in the time domain ${ }^{15}$. Simulations taking into account microscopic and macroscopic effects confirm that each beamlet consists of an isolated attosecond pulse, which hardly experiences any distortion compared to the case where we use the same intensity and pulse duration, but no spatial chirp is applied on the driving laser (Supplementary Sections SI-B, SII).

\section{Temporal measurements by photonic streaking}

Figure 3a shows how the XUV beam spatial profile changes with the carrier-envelope phase (CEP) of the laser pulse (Supplementary Movie S1). As the CEP is scanned, we observe a continuous drift of the propagation angle of all beamlets due to a time shift of the attosecond pulse train under the pulse envelope when CEP changes, which is converted into an angular shift in the presence of $\mathrm{WFR}^{8,9}$. This effect allows CEP fluctuations in the generation medium to be measured directly (Fig. 3b,c), and could be used in the future as a feedback signal to achieve CEP stabilization precisely in this medium. This is also direct evidence of the time-to-angle mapping resulting from WFR. This mapping is the core idea of photonic streaking, and it provides a direct way to access temporal information, as we now demonstrate.

Owing to the evolution of the laser intensity along the driving laser pulse, the properties of the generated attosecond pulses are different in each half-laser-cycle. So far, determining these changes experimentally has required sophisticated techniques or analysis $^{12,13}$. However, they can be measured easily by photonic streaking, as demonstrated in Fig. 2b,c. The maximum energy of the XUV spectrum generated in a given half-cycle (known as a half-cycle cutoff ${ }^{12}$ ) is observed to change from one attosecond pulse to the next (c1-c4 and s1-s4 labels in Fig. 2b,c). As expected, this evolution depends on the CEP. The reason is illustrated by the electron trajectories displayed next to Fig. 2b,c: the half-cycle cutoff is determined by the amplitude of the laser field in the second step of the generation process where the electron is accelerated towards its parent ion. The highest cutoff occurs only once in Fig. 2b, suggesting that a cosine pulse is used for the generation: this is obtained for path ' $c 2$ ', where the recombination occurs at the peak of this cosine pulse. When the CEP is changed by $\pi / 2$, the highest cutoff occurs twice because of paths 's2' and 's3' being driven by a sine pulse. In both cases, the variation of these cutoffs is reproduced well by numerical simulations, as shown by the crosses in Fig. 2b,c (see Supplementary Section SI-C for details).

In contrast to the spectral cutoff, the amplitude of the attosecond pulse is mostly determined by the field amplitude at the time of ionization, which sets the magnitude of the initial electron wave packet. As a result, the most intense attosecond pulses are expected to be emitted in the half-cycle(s) following the highest spectral cutoff(s). This is precisely what is observed in Fig. $2 b, c$, where the spectrally integrated signal plotted in the side panel provides the temporal intensity profile of the attosecond pulse train. Only one most intense pulse is observed for the cosine pulse, for path ' $c 3$ ', while two are emitted for the sine pulse, corresponding to paths ' $\mathrm{s} 3$ ' and ' $\mathrm{s} 4$ '.

If ionization alone were responsible for the different peak amplitudes, they should be equal for the two pulses associated with paths 'c2' and 'c4' in Fig. 2b, as well as for those associated with paths 's3' and 's4' in Fig. 2c, because the ionization rate is almost identical for these pairs of half-cycles. However, considering our short pulse 

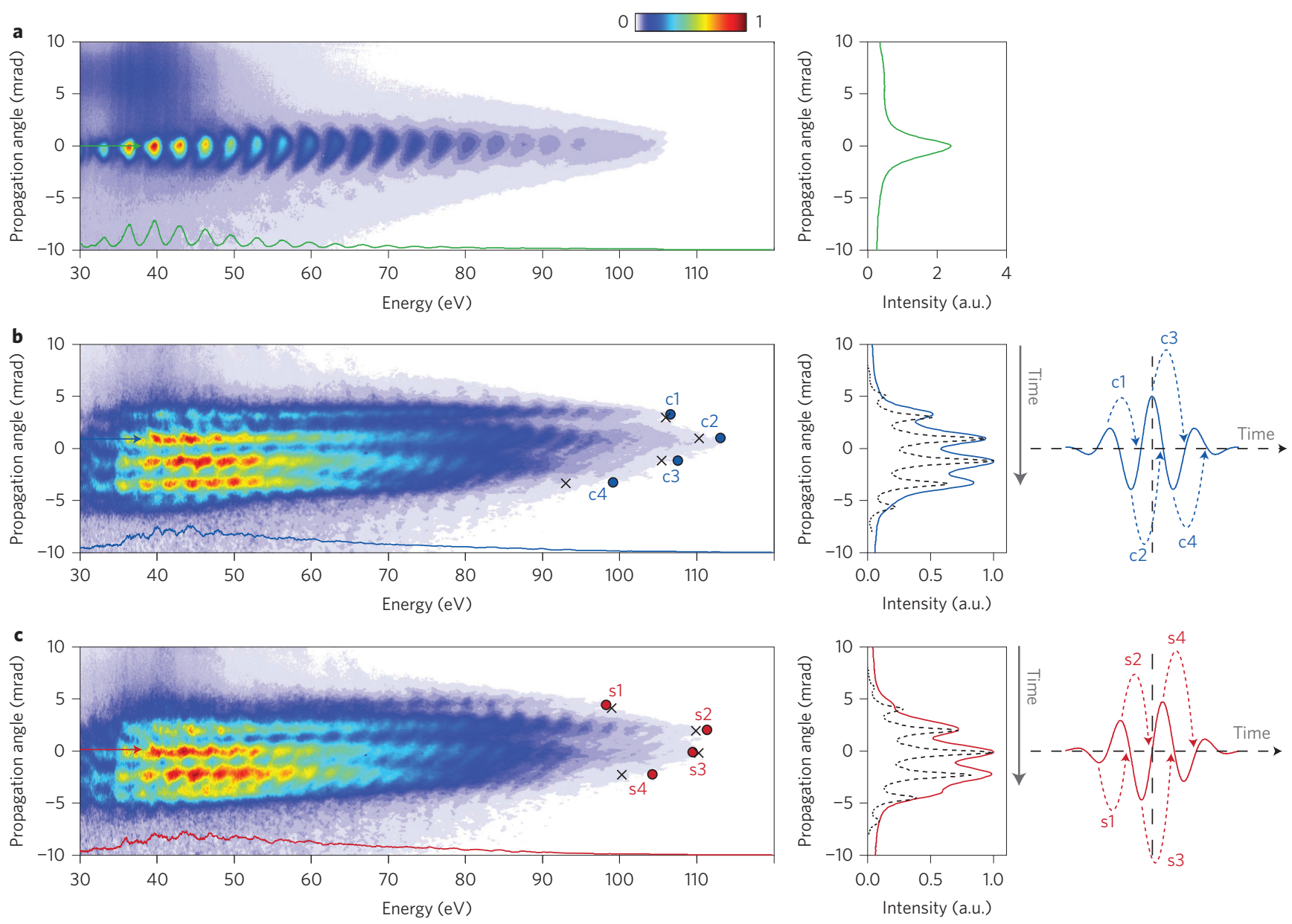

Figure 2 | Harmonic generation in Ne probed by photonic streaking. a-c, Angularly resolved XUV spectrum (colour map) generated in Ne without any spatial chirp of the laser beam (a) and with spatially chirped $\left(\theta=20^{\circ}\right)$ cosine $(\mathbf{b})$ and sine (c) waveforms of the laser pulse. The measured cutoffs of each half-cycle are shown with blue (b) and red (c) circles. At the bottom of each graph, the XUV spectrum at one angle (indicated by the horizontal arrow) is shown by the solid lines. Right panels: spatial profile of the XUV beam, obtained by integrating the angularly resolved XUV spectrum for the entire energy range. The cutoffs and spatial profiles obtained from the calculations are shown by black crosses and black dotted lines. Note that the reduced contrast of the modulations in the experimental spatial profile is due to residual CEP fluctuations in the medium (Fig. 3). The cutoffs of each half cycle are defined as the energies where the spectral intensity drops to $1.5 \%$ of its maximum for both the experiment and the calculation. To the right of $\mathbf{b}$ and $\mathbf{c}$, the process of harmonic generation is illustrated for cosine and sine waveforms of the laser pulse.

duration $(\leq 10 \mathrm{fs})$, non-adiabatic effects also contribute: once ionized, the electron wave packets corresponding to 'c2' and ' $c 4$ ' are driven by significantly different fields, and the corresponding probabilities of recollision with the core are affected. For instance, when the field amplitude decreases between ionization and recollision, in the trailing edge of the pulse, fewer electrons can be brought back to the parent ion by the laser field, and this reduces the amplitude of the emitted attosecond pulse. These non-adiabatic effects, observed experimentally here for the first time, are accounted for in our model, which reproduces fairly well the measured pulse amplitudes, and in particular predicts an asymmetry of the train as shown with dotted lines in the side panels of Fig. 2b,c (Supplementary Section SI-C).

We now turn to a different generation gas, $\mathrm{N}_{2}$, which has a lower ionization potential that allows us to explore different regimes of HHG. Each XUV spectrum shown in Fig. $4 a-c$ is obtained in a single laser shot at a different peak intensity. At the lowest intensity $\left(2.4 \times 10^{14} \mathrm{~W} \mathrm{~cm}^{-2}\right)$ shown in Fig. $4 \mathrm{a}$, the result is analogous to what was observed for $\mathrm{Ne}$ (Fig. 2b,c). However, the divergence of the half-cycle beams is a little larger because the jet is placed closer to the laser beam focus than before, resulting in a greater spatial overlap of beamlets and hence greater spectral modulations. We observe that the energy spacing between the resulting interference fringes is larger at the beginning of the harmonic pulse $\left(2 \omega_{0}=3.6 \mathrm{eV}\right)$ than at the end $\left(2 \omega_{0}=3.1 \mathrm{eV}\right)$, as highlighted in Fig. $4 \mathrm{a}$ by the yellow double-sided arrows. This variation reveals that the time spacing between adjacent attosecond pulses varies across the laser pulse. Such a variation is expected for HHG in gases, and leads to the well-known femtosecond chirp of individual harmonics ${ }^{16,17}$. Photonic streaking enables us to directly follow the associated shift of the pulse train period in time over multiple cycles of the laser pulse. The measured modulation period varies by $0.5 \mathrm{eV}$ over $2 T_{\mathrm{L}}$, which corresponds to a chirp of $0.25 \mathrm{eV} / T_{\mathrm{L}}$, in good agreement with our theoretical estimate of $0.27 \mathrm{eV} / T_{\mathrm{L}}$ (Supplementary Section SI-D).

\section{Ionization gating probed by photonic streaking}

As we have demonstrated by studying the well-understood dynamics of HHG at low intensity, photonic streaking encodes the temporal dynamics of the nonlinear medium onto a spatial dimension. We now apply photonic streaking to the less well understood regime of $\mathrm{HHG}$, where ionization plays a major role. In $\mathrm{N}_{2}$, we can saturate ionization $(>95 \%)$ with a peak intensity of $\sim 5 \times 10^{14} \mathrm{~W} \mathrm{~cm}^{-2}$ for the 8.5 fs pulse duration. As the medium ionization becomes significant, isolated attosecond pulses can be produced through ionization gating ${ }^{18-23}$. Two effects can contribute 

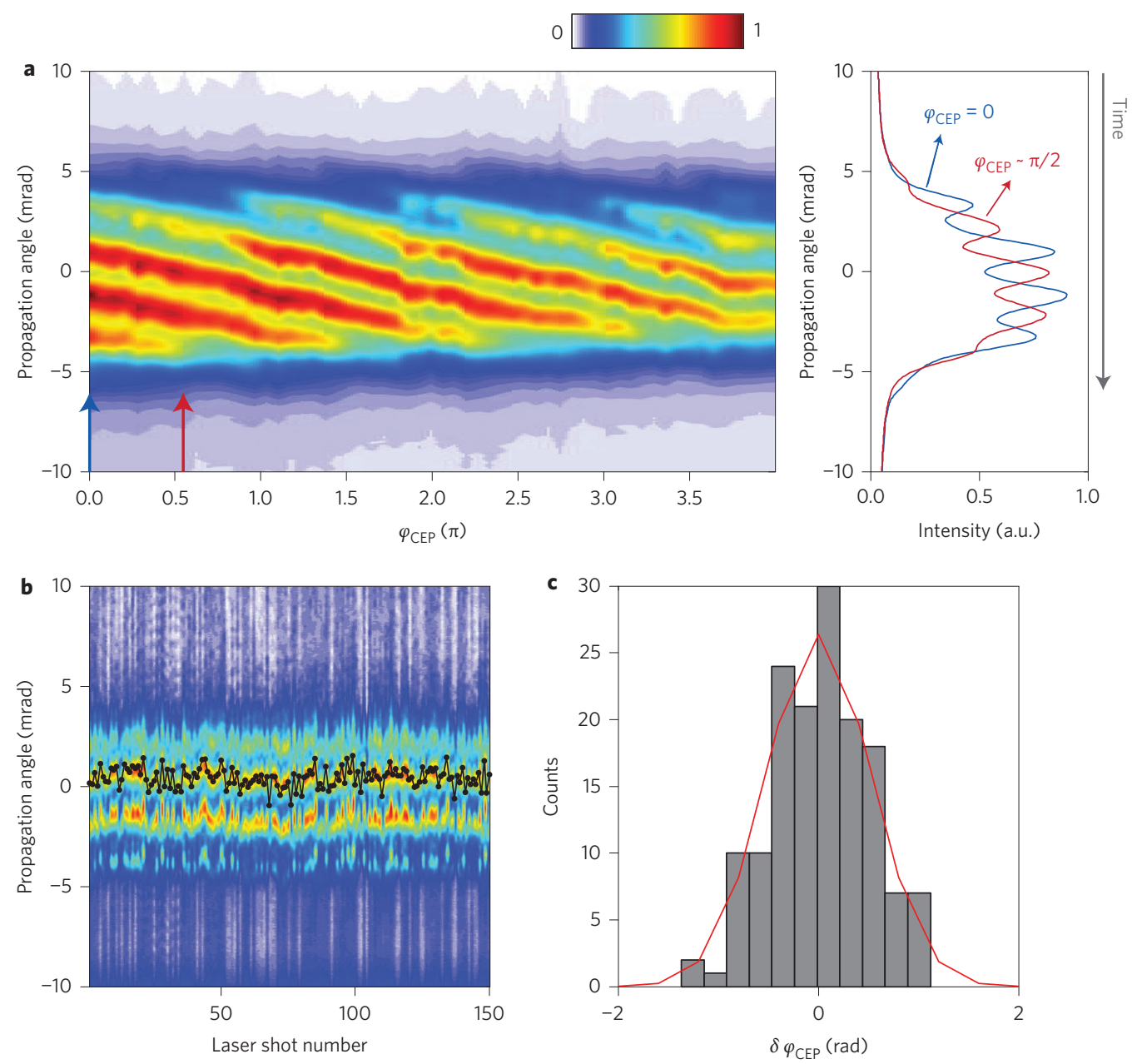

Figure 3 | Time-to-angle mapping in photonic streaking. a, The colour code shows the spatial profile of the XUV beam (averaged over 30 laser shots), in the presence of wavefront rotation, as a function of the CEP of the driving laser pulse. The CEP was varied by slightly translating one wedge of the pair shown in Fig. 1 with respect to the other. Right panel: two lineouts at two particular CEPs differing by $\pi / 2$ (same data as in the right panels of Fig. $2 b, c$ ). The drift of the spatial profile with CEP originates from the drift in time of the emitted attosecond pulse train. $\mathbf{b}, \mathbf{c}$, The colour map (b) shows 150 successive single-shot spatial profiles of the XUV beamlets. The black line with dots shows the shot-to-shot fluctuations of the central position of one of the beamlets, and reveals the residual CEP jitter in the generation medium. This effect provides a direct way of determining the CEP statistics in this medium (c), leading to a standard deviation of the CEP of $\sim 500$ mrad in our experiment.

to ionization gating in the rising part of the laser pulse: (i) groundstate depletion of gas molecules ${ }^{19}$ or (ii) destruction of phase matching by the free electrons ${ }^{20,21}$.

As we increase the intensity of the laser pulse to $3.4 \times 10^{14} \mathrm{~W} \mathrm{~cm}^{-2}$, Fig. $4 \mathrm{~b}$ shows that the angular separation between the central beamlets widens from $2.4 \mathrm{mrad}$ (Fig. 4a) to $3.4 \mathrm{mrad}$ (see beamlets around $0 \mathrm{mrad}$ in Fig. $4 \mathrm{~b}$ ), and the spectral modulation amplitude decreases, while the opposite occurs for the first beamlets. These effects can be attributed to the rapid plasma formation in the fewcycle laser pulse, which results in a time- and space-dependent phase shift $\phi_{l}(t, y, \xi)=\omega_{\mathrm{p}}\left[n_{\mathrm{e}}(t, y, \xi)\right]^{2} \lambda(y) \xi /\left(4 \pi c^{2}\right)$ on the laser field, where $\xi$ is the propagation distance and $\omega_{\mathrm{p}}$ is the plasma frequency. Thus, the wavefronts of the laser beam change depending on both the instantaneous free electron density $n_{\mathrm{e}}(t, y, \xi)$ and the spatially dependent wavelength $\lambda(y)$, making the wavefront rotation time- and space-dependent. This change of the wavefront can modulate the angular separation between successive attosecond pulses compared to the low-intensity case, showing that the timeto-angle mapping, while preserved, does not necessarily remain purely linear in this regime.

As we increase the intensity further to $4.5 \times 10^{14} \mathrm{~W} \mathrm{~cm}^{-2}$, as shown in Fig. 4c, XUV emission beyond $50 \mathrm{eV}$ photon energy only occurs for the first few half-cycles. The angular separation between these beamlets is very narrow, indicating the rapid phase variation at the beginning of the driving laser pulse. The last strong emission near 7 mrad has a continuous spectrum beyond $67 \mathrm{eV}$, as marked with a black arrow. This provides the first experimental evidence that, in this spectral range, a single attosecond pulse is generated in the rising part of the driving pulse, selected by ionization gating. The progressive influence of ionization on the pulse train profile, eventually leading to this single attosecond pulse, is clearly observed on the spatial profiles displayed in the side panels of Fig. 4, obtained by integrating the spatially resolved spectra around the cutoff. In our generation conditions, both depletion and phase matching contribute to this gating. With photonic streaking, the interplay between these two effects can be investigated in the future by varying both the laser intensity and the gas density.

\section{Conclusions}

We have demonstrated a new scheme for ultrafast measurementsphotonic streaking - that can probe the temporal dynamics of HHG in a nonlinear medium with unprecedented simplicity. By applying this technique to HHG in gases, we have thus been able to provide 

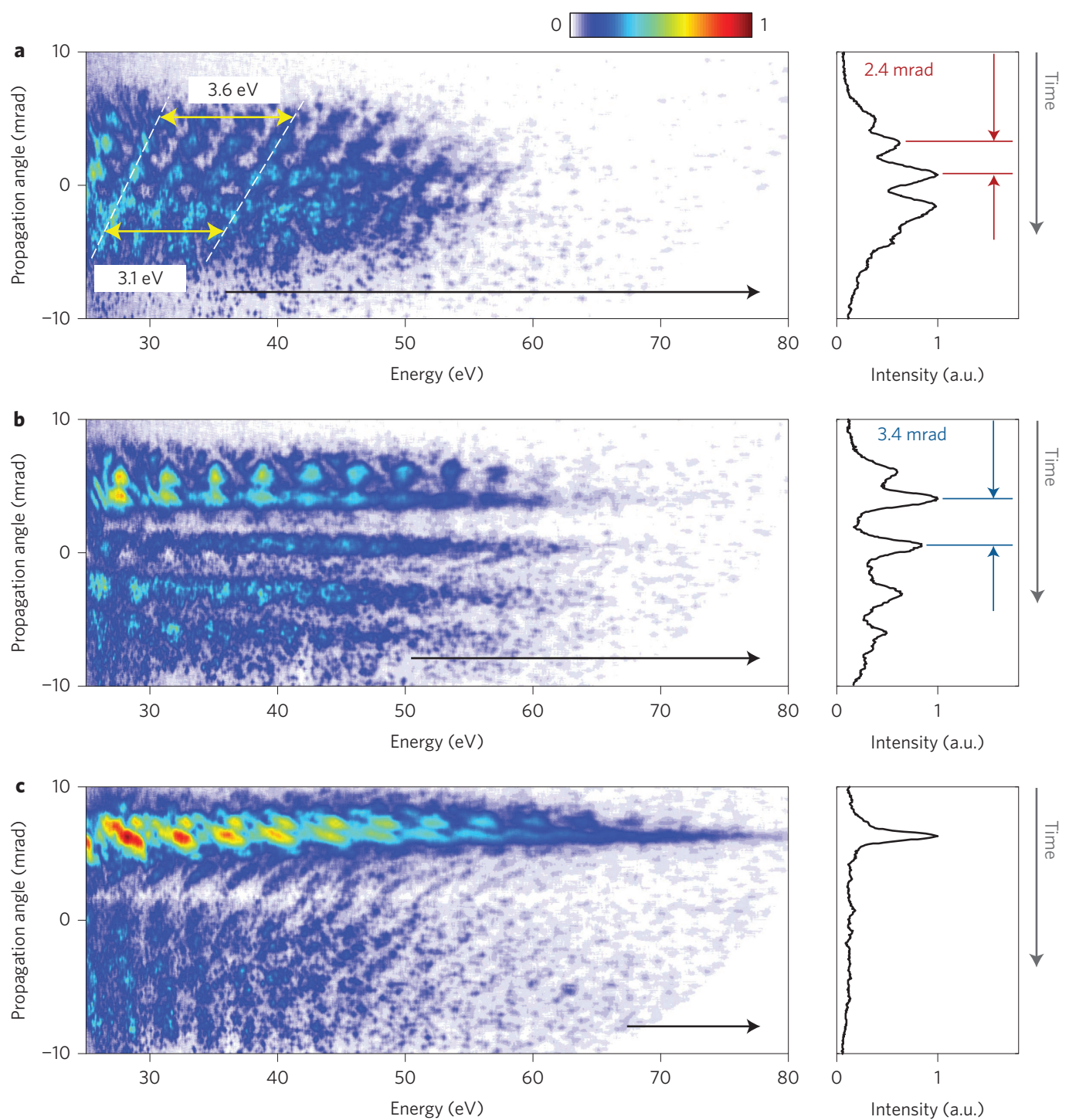

Figure 4 | lonization gating probed by photonic streaking. a-c, Angularly resolved XUV spectra measured in $\mathrm{N}_{2}$ in a single laser shot with a spatial chirp $\left(\theta=20^{\circ}\right)$. The peak intensities of the laser pulses are estimated to be $2.4 \times 10^{14}, 3.4 \times 10^{14}$ and $4.5 \times 10^{14} \mathrm{~W} \mathrm{~cm}^{-2}$ for $\mathbf{a}$, $\mathbf{b}$ and $\mathbf{c}$, respectively. In $\mathbf{a}$, the yellow double-sided arrows show the energy spacings corresponding to six photons, at the beginning and end of the laser pulse, respectively. The energy spacing for two photons $\left(2 \omega_{0}\right)$ changes from $3.6 \mathrm{eV}$ to $3.1 \mathrm{eV}$. The curves in the right panels show the normalized spatial profile of the XUV beam in each case. These profiles were obtained by integrating the angularly resolved XUV spectra around the cutoff, over the energy ranges indicated by the black arrows, which correspond to energies higher than $60 \%$ of the cutoffs expected from the peak intensities of the laser pulse. From the blueshift of the spectral fringes, the change in electron density in the rising part of the laser pulse can be estimated as $\delta_{\mathrm{ne}}=1.4 \pm 0.2 \times 10^{17} \mathrm{~cm}^{-3}$ in the case of Fig. $4 \mathrm{~b}$.

the first direct experimental evidence of the influence of non-adiabatic effects in electron dynamics, and of the restriction of XUV emission to the early part of the laser pulse when ionization becomes significant. More generally, photonic streaking will provide direct access to strong-field dynamics in any HHG system, without the need for pump-probe experiments. It will be a key tool for studying and optimizing the phase-matching response, which is critical for the efficient generation of highenergy $(\sim \mathrm{keV})$ photons $s^{22,24,25}$. It also opens the way to probing the structure ${ }^{2}$ and ultrafast dynamics of polar molecules ${ }^{26}$ in molecular gases, because photonic streaking spatially encodes attosecond pulses generated from opposite recollision directions.

We have also provided the first experimental evidence of an attosecond lighthouse generated in a gas medium, showing that, under appropriate conditions, the general concept proposed in ref. 8 is robust against propagation and phase-matching effects. This approach for isolating attosecond pulses has many advantages over previously demonstrated techniques ${ }^{27-30}$. While retaining the simplicity of spectral filtering at the cutoff $\mathrm{f}^{27}$, it can produce isolated pulses over the full XUV spectral bandwidth, as in the case of polarization gating ${ }^{28}$, but without sacrificing generation efficiency. Like this last technique, it can be combined with other schemes such as $\omega-2 \omega$ mixing to obtain isolated attosecond pulses with longer laser drivers ${ }^{29}$. Such light sources, delivering a collection of beamlets consisting of isolated attosecond pulses, provide a natural scheme for attosecond pump-probe experiments, and should therefore become a major asset for the development of attosecond science.

\section{Methods}

For the experiments, we used a CEP-stabilized laser pulse post-compressed to $5 \mathrm{fs}$ (without spatial chirp) in a Ne-filled hollow-core fibre. A spatial chirp is imposed at the focus by rotating one wedge of the pair (BK7, $8^{\circ}$ wedge angle). The duration of the laser pulse at the focus increases when the spatial chirp is imposed. From the variation of the half-cycle cutoffs shown in Fig. 2b,c, we estimate the duration of the spatially chirped laser pulse to be $8.5 \mathrm{fs}$ at the focus. The vertical beam size (FWHM) is also increased from $32 \mu \mathrm{m}$ (without spatial chirp) to $42 \mu \mathrm{m}$ (with spatial chirp). The laser beam is focused by a spherical mirror with an 
$f$-number of 45 , leading to a Rayleigh length of $z_{\mathrm{R}}=12 \mathrm{~mm}$ on the gas jet placed at 2-3 mm before the focus (Ne shown in Fig. 2) and at the focus $z=0\left(\mathrm{~N}_{2}\right.$ shown in Fig. 4). The peak intensity of the laser pulse is deduced from the measured cutoffs in Fig. 2 and Fig. 4a. For Fig. 4 b,c, the peak intensity is deduced from that in Fig. $4 \mathrm{a}$ by considering the change in laser pulse energy. The nozzle diameter of the gas jet is $250 \mu \mathrm{m}$ with a backing pressure of 4 bar. A vertical slit is located $30 \mathrm{~cm}$ from the gas jet, with its direction parallel to the plane of angular dispersion of the attosecond lighthouse. The angularly resolved XUV spectrum is horizontally dispersed in wavelength with a variable-line-spacing grating, and recorded by a microchannel plate coupled to a phosphor screen imaged by a charge-coupled device camera.

Received 9 January 2013; accepted 29 May 2013; published online 7 July 2013

\section{References}

1. Corkum, P. B. Plasma perspective on strong field multiphoton ionization. Phys. Rev. Lett. 71, 1994-1997 (1993).

2. Itatani, J. et al. Tomographic imaging of molecular orbitals. Nature 432, 867-871 (2004)

3. Wörner, H. J. et al. Conical intersection dynamics in $\mathrm{NO}_{2}$ probed by homodyne high-harmonic spectroscopy. Science 334, 208-212 (2011).

4. Antoine, P., L'Huillier, A. \& Lewenstein, M. Attosecond pulse trains using high-order harmonics. Phys. Rev. Lett. 77, 1234-1237 (1996).

5. Paul, P. M. et al. Observation of a train of attosecond pulses from high harmonic generation. Science 292, 1689-1692 (2001).

6. Niikura, H. et al. Sub-laser-cycle electron pulses for probing molecular dynamics. Nature 417, 917-922 (2002).

7. Baker, S. et al. Probing proton dynamics in molecules on an attosecond time scale. Science 312, 424-427 (2006).

8. Vincenti, H. \& Quéré, F. Attosecond lighthouses: how to use spatiotemporally coupled light fields to generate isolated attosecond pulses. Phys. Rev. Lett. 108, 113904 (2012).

9. Wheeler, J. A. et al. Attosecond lighthouses from plasma mirrors. Nature Photon. 6, 829-833 (2012).

10. Akturk, S., Gu, X., Gabolde, P. \& Trebino, R. The general theory of first-order spatio-temporal distortions of Gaussian pulses and beams. Opt. Express 13, 8642-8661 (2005).

11. Krausz, F. \& Ivanov, M. Y. Attosecond physics. Rev. Mod. Phys. 81, 163-234 (2009).

12. Haworth, C. et al. Half-cycle cutoffs in harmonic spectra and robust carrierenvelope phase retrieval. Nature Phys. 3, 52-57 (2006).

13. Kim, K. T., Ko, D. H., Park, J. J., Tosa, V. \& Nam, C. H. Complete tempora reconstruction of attosecond high-harmonic pulse trains. New J. Phys. 12, 083019 (2010).

14. Lewenstein, M., Balcou, P., Ivanov, M. Y., L'Huillier, A. \& Corkum, P. B. Theory of high-harmonic generation by low-frequency laser fields. Phys. Rev. A 49, 2117-2132 (1994).

15. Chini, M., Wang, H., Khan, S. D., Chen, S. \& Chang, Z. Retrieval of satellite pulses of single isolated attosecond pulses. Appl. Phys. Lett. 94, 161112 (2009)

16. Shin, H. J., Lee, D. G., Cha, Y. H., Hong, K. H. \& Nam, C. H. Generation of nonadiabatic blueshift of high harmonics in an intense femtosecond laser field. Phys. Rev. Lett. 83, 2544-2547 (1999).
17. Varjú, K. et al. Reconstruction of attosecond pulse trains using an adiabatic phase expansion. Phys. Rev. Lett. 95, 243901 (2005).

18. Christov, I. P., Murnane, M. M. \& Kapteyn, H. C. High-harmonic generation of attosecond pulses in the 'single-cycle' regime. Phys. Rev. Lett. 78, 1251-1254 (1997).

19. Kim, K. T., Kim, C. M., Baik, M. G., Umesh, G. \& Nam, C. H. Single sub-50attosecond pulse generation from chirp-compensated harmonic radiation using material dispersion. Phys. Rev. A 69, 051805 (2004).

20. Abel, M. et al. Isolated attosecond pulses from ionization gating of high-harmonic emission. Chem. Phys. 366, 9-14 (2009).

21. Ferrari, F. et al. High-energy isolated attosecond pulses generated by above-saturation few-cycle fields. Nature Photon. 4, 875-879 (2010).

22. Popmintchev, T. et al. Bright coherent ultrahigh harmonics in the keV X-ray regime from mid-infrared femtosecond lasers. Science 336, 1287-1291 (2012).

23. Sansone, G., Poletto, L. \& Nisoli, M. High-energy attosecond light sources. Nature Photon. 5, 655-663 (2011).

24. Geissler, M., Tempea, G. \& Brabec, T. Phase-matched high-order harmonic generation in the nonadiabatic limit. Phys. Rev. A 62, 033817 (2000).

25. Tempea, G., Geissler, M., Schnürer, M. \& Brabec, T. Self-phase-matched high harmonic generation. Phys. Rev. Lett. 84, 4329-4332 (2000).

26. Frumker, E. et al. Oriented rotational wave-packet dynamics studies via high harmonic generation. Phys. Rev. Lett. 109, 113901 (2012).

27. Goulielmakis, E. et al. Single-cycle nonlinear optics. Science 320, 1614-1617 (2008).

28. Sansone, G. et al. Isolated single-cycle attosecond pulses. Science 314, 443-446 (2006).

29. Mashiko, H. et al. Double optical gating of high-order harmonic generation with carrier-envelope phase stabilized lasers. Phys. Rev. Lett. 100, 103906 (2008).

30. Goulielmakis, E. et al. Real-time observation of valence electron motion. Nature 466, 739-743 (2010).

\section{Acknowledgements}

The authors acknowledge funding from the Natural Sciences and Engineering Research Council, the Air Force Office of Scientific Research and the National Research Council-Commissariat à l'énergie atomique et aux énergies renouvelables agreement. F.Q. acknowledges support from the European Research Council (ERC grant agreement no. 240013).

\section{Author contributions}

K.T.K., D.M.V., P.B.C. and F.Q. conceived the idea and designed the experiment.

K.T.K., C.Z., T.R. and J.-F.H. performed the experiment and collected the data. K.T.K. and T.A. provided the numerical analysis. All authors contributed in analysing the experimental data and writing the manuscript.

\section{Additional information}

Supplementary information is available in the online version of the paper. Reprints and permissions information is available online at www.nature.com/reprints. Correspondence and requests for materials should be addressed to K.T.K. and F.Q.

\section{Competing financial interests}

The authors declare no competing financial interests. 

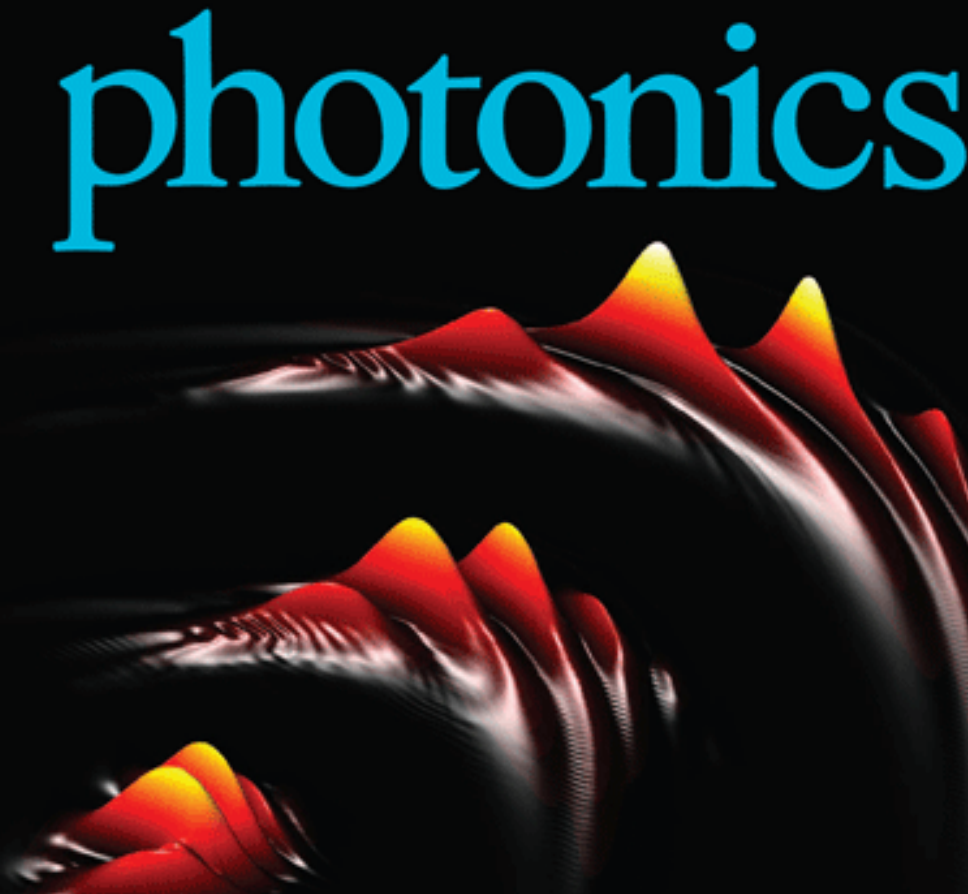

SOLITONS

The weakest interactions

ORGANIC OPTOELECTRONICS Detecting the chirality of light QUANTUM ENTANGLEMENT Enhanced cat-state spectroscopy

\section{Ultrafast photonic streaking}




\title{
Supplementary Information on \\ Photonic Streaking of Attosecond Pulse Trains
}

\author{
Kyung Taec Kim, ${ }^{1}$ Chunmei Zhang, ${ }^{1}$ Thierry Ruchon, ${ }^{2}$ Jean-François Hergott, ${ }^{2}$ \\ Thierry Auguste, ${ }^{2}$ D. M. Villeneuve, ${ }^{1}$ P. B. Corkum, ${ }^{1}$ and Fabien Quéré ${ }^{2}$ \\ ${ }^{1}$ Joint Attosecond Science Laboratory, \\ National Research Council and University of Ottawa, \\ 100 Sussex Drive, Ottawa ON K1A OR6, Canada \\ ${ }^{2}$ CEA, IRAMIS, Service des Photons Atomes et Molécules, \\ F-91191 Gif-sur-Yvette, France
}

In this supplementary information, we provide a more detailed numerical analysis of photonic streaking and of the different effects discussed in the manuscipt. To this end, we use two different theoretical approaches.

In Sec. I, we use a simple model for high harmonic generation. XUV radiation is calculated using atoms distributed in 2-D space along the vertical and propagation directions. We assume Gaussian envelopes in space and time for the driving laser pulse. The spatial chirp of the laser pulse is described by changing the central wavelength along the vertical direction in the medium. The single atom dipole moments are calculated, both in amplitude and phase, by using the strong field approximation (SFA) [1] with the Yudin-Ivanov ionization model [2] for two main (short and long) quantum paths. The interference between these dipoles, leading to the macroscopic build-up of the harmonic signal, is accounted for. However, the propagation effects of the laser and XUV pulses in the medium are not taken into account: these propagations are the same as in vacuum (which includes the Gouy phase). As a result, the effect of plasma formation, that can deform the laser pulse itself, is not considered. The refractive index of the gas medium is not taken into account either. This simple model provides a computationally efficient way to analyse effects of photonic streaking. The experimental observations are very well reproduced when ionization is negligible. We use this simple model in Sec. I to support the arguments that we made in the main text about the contributions of the different quantum paths (Sec. I A), the space-time couplings of attosecond pulses generated by WFR (Sec. IB), the time structure of the pulse trains 
generated with different CEP's (Sec. IC) and the temporal chirp of the harmonics (Sec. ID). All these effects are analyzed in the presence of WFR, leading to photonic streaking.

In Sec. II, we use a more sophisticated simulation code. In this case, all effects are taken into account, including the full propagation of both the laser and XUV field in the ionized medium. Since we consider a spatially chirped laser pulse for high harmonic generation, one might think that this space-time coupling of the laser pulse could destroy the temporal structure of the attosecond pulses along the propagation. In order to show that the temporal profile of the attosecond pulse is preserved after the propagation, we use this more elaborated code, which is described more precisely in Sec. II.

\section{PHOTONIC STREAKING CALCULATED BY A SIMPLE MODEL}

\section{A. Quantum path analysis of XUV radiation with photonic streaking}

XUV radiation obtained through high harmonic generation originates from two main quantum paths -long and short paths. In order to see how these two quantum paths contribute to the XUV spectra observed at the far-field, we calculate four different XUV spectra as shown in Fig. S1. First, the XUV radiation is calculated using a zero-length medium with an artificial temporal window that allows the ionization only for short quantum paths in one half optical cycle near the peak of the laser pulse. This allows us to see only one beamlet at the far-field in which only short quantum paths contribute as shown in Fig. S1a. Second, the duration of the artificial window is increased so that both short and long quantum paths are allowed as shown in Fig. S1b. Short trajectory components are concentrated near a propagation angle of $1 \mathrm{mrad}$, while long trajectory components are diverging more. XUV radiation generated through these two paths interfere, leading to spatio-spectral fringes. Third, XUV radiation is calculated using a zero-length medium without any artificial temporal window as shown in Fig. S1c. The diverging long trajectory components of each beamlet overlap with other beamlets, showing very complicated XUV spectrum. Finally, XUV spectrum is calculated with a $250-\mu \mathrm{m}-\mathrm{long}$ medium as shown in Fig. S1d. Since the long trajectory components are more sensitive to the phase and intensity variation of the laser pulse along the propagation direction, they almost disappear due to the poor phase matching when the medium length is taken into account in the calculation. The calculated 
XUV spectrum (Fig. S1d) well reproduces the one observed in the experiment, showing that the short quantum paths dominantly contribute to the beamlets.
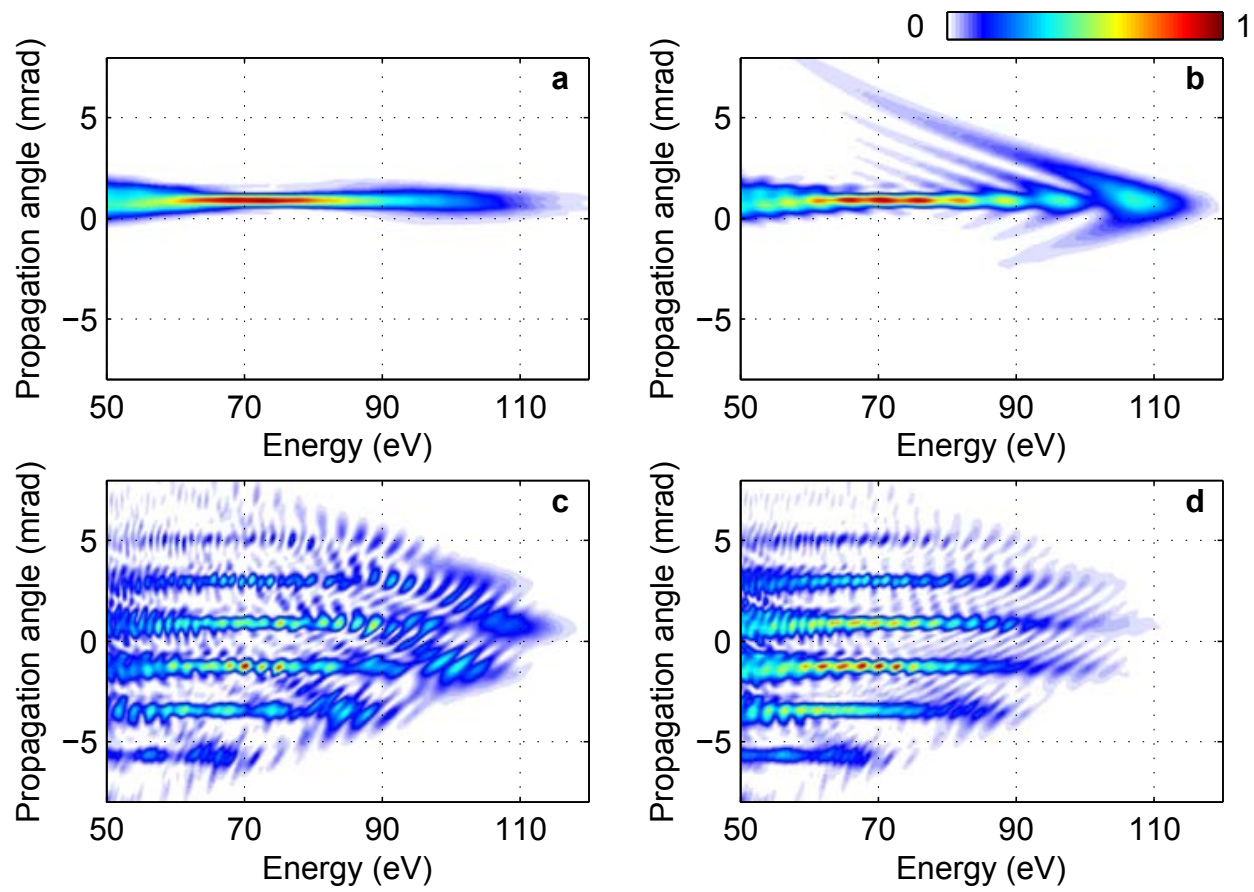

FIG. S1: XUV spectra calculated at the far-field. The far-field XUV spectra obtained from Ne are calculated using a $8.5 \mathrm{fs}$ Gaussian laser pulse with an intensity of $4.8 \times 10^{14} \mathrm{~W} / \mathrm{cm}^{2}$. The central wavelength of the laser beam varies from $680 \mathrm{~nm}$ to $850 \mathrm{~nm}$ across the beam size of $40 \mu \mathrm{m}$ full width at half maximum (FWHM). a, XUV spectrum calculated with zero medium length. An artificial temporal window that allows the ionization only for short quantum paths in one half optical cycle is applied to see only one beamlet. $\mathbf{b}$, The same XUV spectrum as in $\mathbf{a}$, but both short and long quantum path are considered in one half optical cycle. c-d, XUV radiation calculated with (c) zero medium length and (d) 250- $\mu$ m-long medium. No artificial temporal window is applied.

\section{B. Space-time coupling of attosecond pulses produced by photonic streaking}

Attosecond pulses generated by spatially chirped laser pulses can be used as a light source for ultra-fast pump-probe experiments. For those experiments, the attosecond pulses are refocused on a second target, e.g. to produce photo-electrons or ions. In this section, we compare temporal profiles of the attosecond pulses calculated with and without wave front rotation (WFR) of the laser beam, to see the possible distortion (space-time couplings) induced by WFR on the attosecond pulses at the second target.

The temporal profiles of the attosecond pulses at the second target are shown after the 
spectral filtering from $50 \mathrm{eV}$ to $80 \mathrm{eV}$ in Fig. S2a and b. In Fig. S2b, the temporal profiles of the attosecond pulses progressively get tilted for each half cycle. One would think this makes the duration of the attosecond pulse longer. However, each pulse propagates in different directions. When it is viewed along its own propagation direction, the duration of each pulse is not different from the duration of the pulse obtained without WFR. We compare the pulse profile averaged along the vertical direction near 1 fs that propagates on axis (near $y=0$ ) as shown in Fig. S2c. The pulse shapes are almost identical and their durations are similar (140 \pm 1 as). This shows that the space-time coupling of the isolated attosecond pulse induced by the spatial chirp of the laser pulse is negligible.
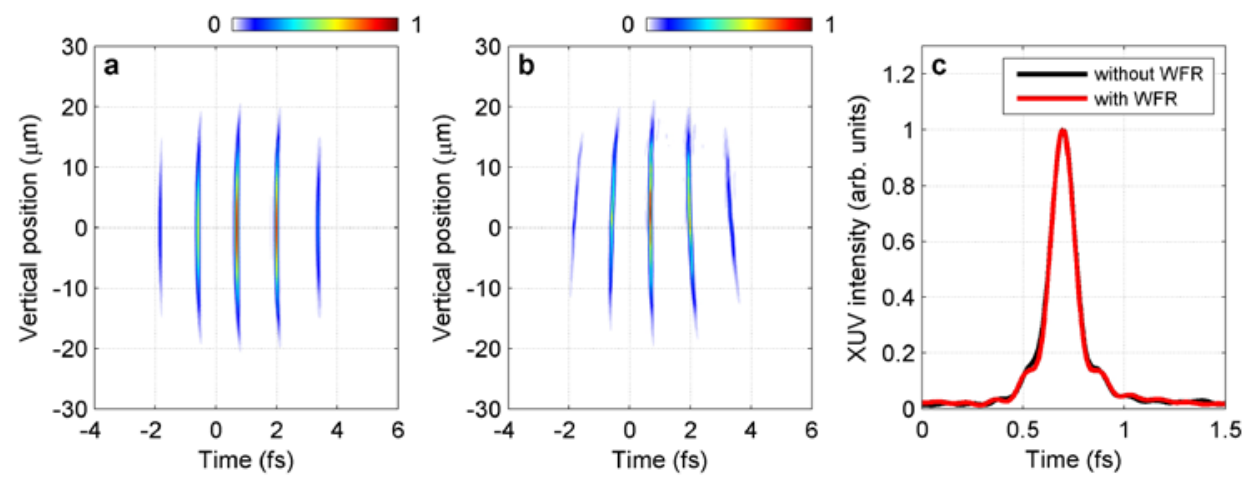

FIG. S2: Intensity profiles of the attosecond pulses at the second target calculated with and without spatial chirp of the driving laser. The XUV radiations are calculated for Ne driven by a 8.5 fs Gaussian sine laser pulse with an intensity of $4.8 \times 10^{14} \mathrm{~W} / \mathrm{cm}^{2}$. Spectral filtering from $50 \mathrm{eV}$ to $80 \mathrm{eV}$ is applied. a, Intensity profile (color code) of the attosecond pulses generated without spatial chirp as a function of the vertical position at the second target. The central wavelength of the laser pulse is fixed to be $765 \mathrm{~nm}$. b, Intensity profile (color code) of the attosecond pulses generated with spatial chirp as a function of the vertical position at the second target. The wavelength of the laser pulse varies from $680 \mathrm{~nm}$ to $850 \mathrm{~nm}$. c, The spatially-integrated intensity profiles of the attosecond pulse in the middle of the train obtained from (a) and (b).

\section{XUV spectra calculated with cosine and sine laser pulses}

The spectral features of the XUV radiation produced through high harmonic generation, such as cut-off energies and XUV intensities of each half cycle, are different depending on the instantaneous laser intensity. In this section, we compare the single atom responses obtained by cosine and sine laser pulses. We also show the far-field XUV spectra calculated with cosine and sine laser pulses in the presence of WFR, to demonstrate how photonic 
streaking can be used to map these spectral features.

The high harmonic generation process can be described by the three step model; ionization, acceleration, and recombination. The process occurs over two half-cycles of the fundamental laser pulse for short quantum paths. In the first half cycle of the laser field, the electron is liberated and it moves away. At the second half cycle of the laser field, the electron is driven back and recombines with its parent ion, leading to the generation of an XUV photon whose energy corresponds to the sum of the kinetic energy of the electron and the ionization potential of the atom. The maximum energy of the XUV radiation - cutoff is determined by the amplitude of the second half cycle where the electron gains its kinetic energy.

Figure S3a shows the energy of the XUV radiation as a function of recombination time calculated by a single atom SFA model [1]. The maximum energies of the XUV radiation emitted at each half cycle - half cycle cutoffs - are 100.5, 104.7, 98.9, 85.1 eV for paths c1 to $\mathrm{c} 4$, respectively, showing one stronger peak for path 'c2' because a cosine laser pulse is used for high harmonic generation. On the other hand, the cut offs are 95.0, 103.9, 103.0, 92.7 for path s1 to s4, respectively, showing two higher energies for the sine laser pulse as shown in Figure S3b. The calculation confirms that the cutoff energy is determined by the intensity of the half cycle where the recombination occurs.

While the half cycle cut-off is determined by the field amplitude where the recombination occurs, the XUV pulse intensity is mainly determined by the intensity of the half cycle where the ionization takes place. The ionization, the first step of the high harmonic generation, is very sensitive to intensity variation of few cycle laser pulses. The strong ionization occurs only near the peak of the laser pulse as shown with blue lines in Fig. S3c-d. Since the ionization rate at the peak of the laser pulse is much stronger than others, the strongest XUV signal should appear through path 'c3' for cosine and 's3' and 's4' for sine laser pulses. For the accurate analysis on the XUV intensities, however, electron trajectories also need to be taken into account.

The electron can be ionized at any time as shown in Fig. S3c-d. However, the electron ionized early in each half optical cycle (before the local maximum of the laser field) does not recombine to its parent ion. For example for path 'c2', the electron ionized from -0.75 $T_{L}$ to $-0.5 T_{L}$ does not recombine with its parent ion. The electron ionized from $-0.50 T_{L}$ to $-0.45 T_{L}$ (shaded with a grey colour) recombines to the ion through the long quantum path. 

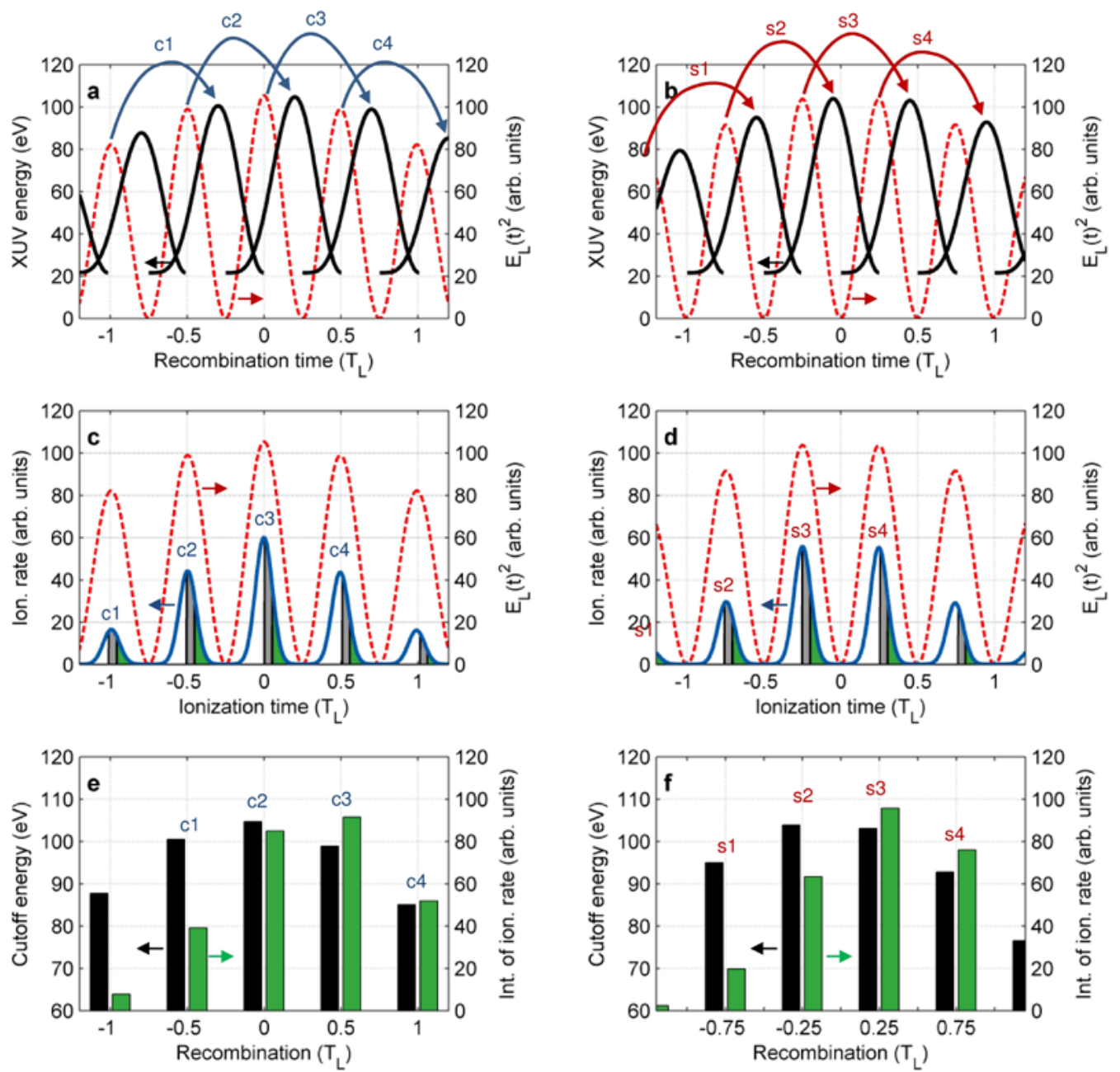

FIG. S3: Spectral features obtained from a Ne atom with cosine and sine laser pulses. The XUV energy and ionization rate are calculated for cosine and sine 8.5-fs Gaussian laser pulses with an intensity of $4.8 \times 10^{14} \mathrm{~W} / \mathrm{cm}^{2}$. The single atom response is calculated by the strong field approximation. The ionization rate is calculated by the Yudin-Ivanov model. $\mathbf{a}-\mathbf{b}$, Energies of XUV radiation as a function of recombination time for cosine (a) and sine (b) laser pulse $E_{L}(t)$. XUV energies are shown with black lines as a function of recombination time. $E_{L}(t)^{2}$ is shown with red dashed line. c-d, Ionization rate for cosine (c) and sine (d) laser pulses. The electron population that recombines through long quantum paths is shaded with a grey colour. The one that recombines through the short quantum path is shaded with a green colour. e-f, Half cycle cut-offs and population of recombining electron for cosine (e) and sine (f) laser pulse. The amount of recombining electron is estimated by integrating the ionization rate for short quantum paths (i.e. green areas for each half cycle) in c and d.

The electron ionized from $-0.45 T_{L}$ to $-0.25 T_{L}$ (shaded with a green colour) recombine through short quantum paths. As we show in the Sec. IA, these short quantum paths mainly contribute to the XUV radiation when the phase matching is taken into account.

Although the ionization rate is almost identical for 'c2' and 'c4' as shown in Fig. S3b, 
their electron trajectories are different due to the intensity of the laser pulse where the recombination takes place. For the path ' $\mathrm{c} 2$ ', the recombination takes place at the peak of the laser pulse. The green area - where the recombination occurs through short trajectory - under the ionization rate is larger. For the path ' $c 4$ ', the recombination occurs when the laser field is weak. So, the green area is smaller. Thus, the analysis of the quantum path explains why the XUV intensity is stronger for the rising part of the laser pulse.

These results are summarized in Fig. S3e-f for cosine and sine laser pulses. The cut-off energies of XUV radiation generated at each half cycle are shown with black bar graphs, showing that the cutoff follows the intensity of the laser pulse at each half cycle. The electron population that recombines with the parent ion through the short trajectory is estimated by calculating the green area in Fig. S3c and d. The result show that the maximum XUV intensity is obtained a half cycle later than the half cycle where the maximum cutoff obtained. Also, the XUV intensity is stronger for the rising part of the laser pulse than the falling part.

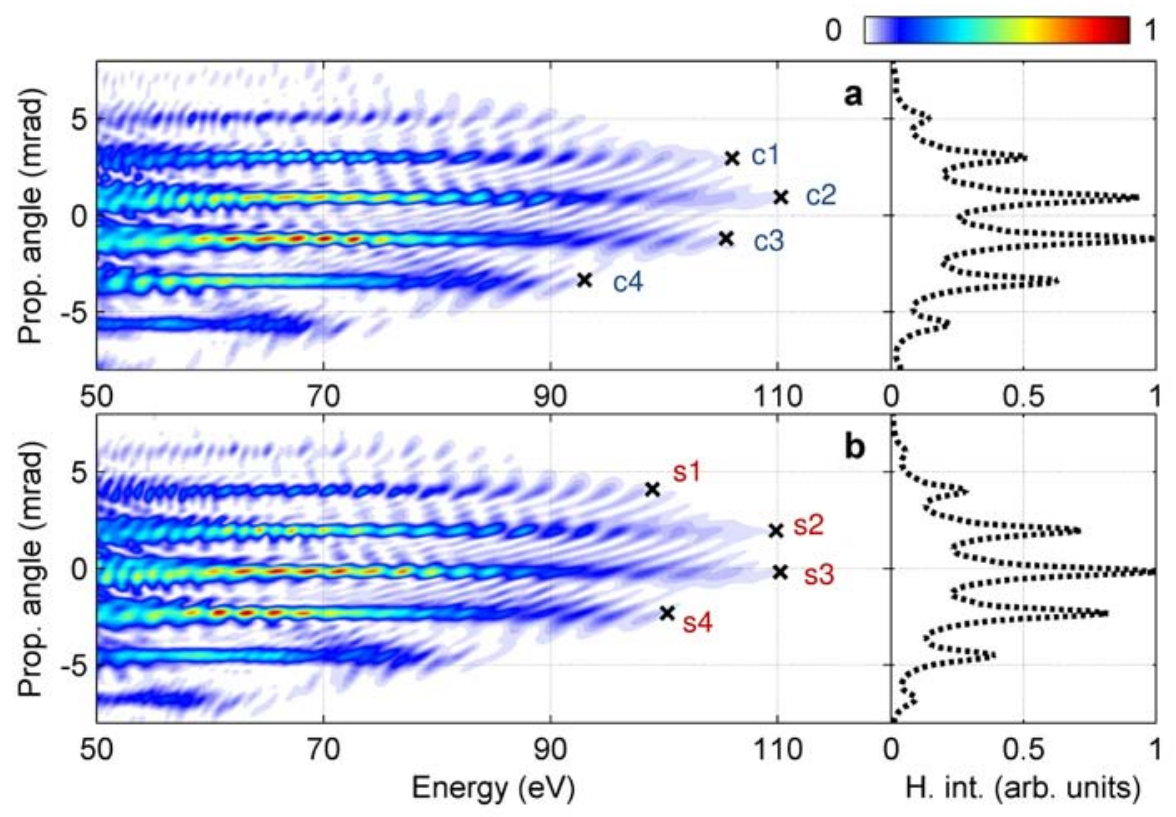

FIG. S4: XUV spectra calculated with cosine and sine laser pulses at the far-field. The XUV spectra obtained from 250- $\mu$ m-long Ne medium are calculated using a 8.5-fs Gaussian laser pulse with an intensity of $4.8 \times 10^{14} \mathrm{~W} / \mathrm{cm}^{2}$. The central wavelength of the laser beam varies from $680 \mathrm{~nm}$ to $850 \mathrm{~nm}$ across the beam size of $40 \mu \mathrm{m}$ FWHM. a-b, XUV spectrum calculated with cosine (a) and sine (b) laser pulses. The black crosses show the cut-off of each half cycle where the XUV intensity is $1.5 \%$ of the maximum XUV intensity. In the right panel, the XUV signals integrated along the horizontal direction are shown with a dotted line. 
In order to see how photonic streaking maps cut-offs and XUV intensities generated at each half cycle at the far-field, we show XUV spectra calculated with cosine and sine laser pulses in Fig. S4. These should be compared with the experimental measurements presented in Fig.2 of the main text. For a cosine laser pulse shown in Fig. S4a, the highest cut-off is obtained at a propagation angle of $1 \mathrm{mrad}$. This is the beamlet for which the recombination occurs at the peak of the laser pulse. In the right panel, the horizontal integrations of XUV radiation are shown with dotted lines. The maximum signal is obtained at -1 mrad for which the ionization takes place at the peak of the laser pulse. For a sine laser pulse shown in Fig. S4b, there are two maximum cut-offs. The maximum XUV intensity is also observed half optical cycle later than the first maximum cut-off. These cut-offs (black crosses) and the XUV intensity (black dotted lines) are shown along with the experimental results in Fig. 2 of the main text.

\section{Blue- and red-shift of the XUV radiation}

XUV bursts are generated in every half optical cycle of the laser pulse. Due to the intensity variation along the laser pulse, the time interval between successive XUV bursts of a given frequency varies. This time interval is shorter than a half optical cycle $\mathrm{T}_{\mathrm{L}} / 2 \mathrm{during}$ the rising part of the pulse envelope, while it is longer than $\mathrm{T}_{\mathrm{L}} / 2$ in the falling part.

In the frequency domain, this variation leads to what is known as the femtosecond chirp of individual harmonics [3]. In the experiment, this chirp is determined by measuring the evolution, along the laser pulse, of the period of the spectral modulations resulting from the overlap of adjacent beamlets. According to Fig. 3a of the main text, this energy interval varies by $0.5 \mathrm{eV}$ over $2 \mathrm{~T}_{\mathrm{L}}$, corresponding to a chirp of $0.25 \mathrm{eV} / \mathrm{T}_{\mathrm{L}}$. We have calculated the emission time of the XUV radiation generated in $N_{2}$ with an intensity of $2.4 \times 10^{14} \mathrm{~W} / \mathrm{cm}^{2}$ at the photon energy of $35 \mathrm{eV}$, as shown in Fig. S5. The interval in emission time changes from $0.451 \mathrm{~T}_{\mathrm{L}}$ to $0.554 \mathrm{~T}_{\mathrm{L}}$ between the beginning and the end of the pulse, corresponding to an harmonic frequency that varies from $3.59 \mathrm{eV}$ to $2.93 \mathrm{eV}$. The calculated chirp $\left(0.67 \mathrm{eV} / 2.5 \mathrm{~T}_{\mathrm{L}}=0.27 \mathrm{eV} / \mathrm{T}_{\mathrm{L}}\right)$ is thus in good agreement with the experimental observation. 


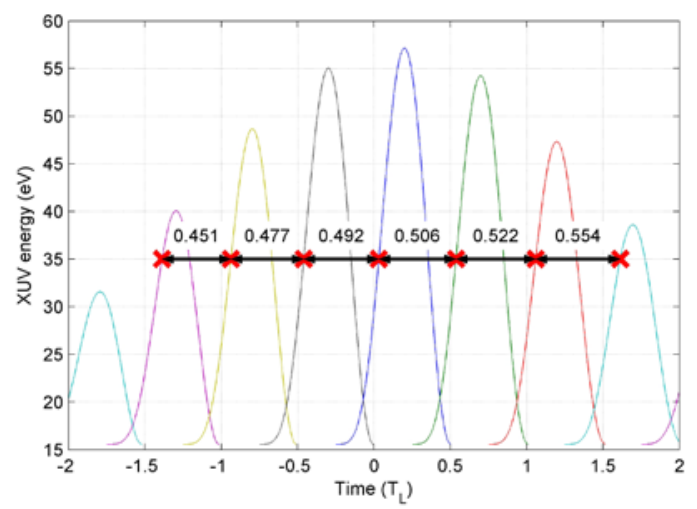

FIG. S5: Emission time of the XUV radiation calculated for $N_{2}$ atoms with a 8.5 fs Gaussian laser pulse. XUV photon energies are shown with curves for every half cycle as a fuction of the emission time. The emission times for $35 \mathrm{eV}$ photon are marked with red crosses. The time intervals between successive emissions, in units of $T_{L}$, are shown above the arrows.

\section{FULL PROPAGATION CALCULATION OF XUV RADIATION WITH PHOTONIC STREAKING}

For simulating the macroscopic contribution of the gas target, we developed a twodimensional (2D) non-adiabatic model in which the deformation of the laser pulse in the ionizing medium is included. We solve the wave equation for the fundamental and the harmonic fields in the slowly evolving wave and paraxial approximations. Note that reducing the problem to $2 \mathrm{D}$ allows for drastically saving computational time without losing the generality of the model. A detailed description of the approach used in this code is given in Refs $[4,5]$. We briefly outline here the main steps underlying the model.

We first solve the wave equation on the fundamental field, which is then used for calculating the source term in the wave equation on the harmonic field. The atomic dipole moment entering the source term is calculated in the strong field approximation, using the model described in Ref. [6]. The optical-field-ionization of the gas, responsible for the depletion of the ground state atom as well as the refraction of the fundamental field and the electron dispersion on the harmonic field, is calculated with Ammosov-Delone-Krainov (ADK) tunneling rates [7]. Atomic dispersion and absorption of the gas (as a function of wavelength) are also taken into account in the wave equation on the harmonic field for photon energies greater than $10 \mathrm{eV}$ [8]. The coupled wave equations are solved in the frame moving with the laser pulse, using a finite-difference scheme in space. Time derivatives are eliminated from 
the equations by a Fourier transform of the fields. We assume an initial fundamental field, with a spatial chirp $\xi$, given at focus by:

$$
E(y, t)=E_{0}(y, t) \times \cos [\phi(y, t)]
$$

with

$$
E_{0}(y, t)=\sqrt{\frac{2 I_{0}}{\epsilon_{0} c}} \times \exp \left\{-\frac{y^{2}}{w_{0}^{2}\left[1+(\xi R / \tau)^{2}\right]}\right\} \times \exp \left\{-\frac{t^{2}}{\tau^{2}\left[1+(\xi R / \tau)^{2}\right]}\right\}
$$

and

$$
\phi(y, t)=\omega_{0} t+\phi_{0}+\frac{-2 \xi R y t}{w_{0} \tau^{2}\left[1+(\xi R / \tau)^{2}\right]} .
$$

$E_{0}(y, t)$ and $\phi(y, t)$ are the space and time varying field envelope and phase, respectively. $w_{0}=\frac{\lambda_{0} f}{\pi R}$ is the beam waist at focus. $R, \tau, f, \lambda_{0}, y$ and $t$ denote the beam waist of the incident beam on the focusing optics, the (unchirped) pulse duration, the focal length of the focusing optics, the fundamental wavelength, the transverse coordinate and the time. $I_{0}$ is the peak intensity at focus, $\omega_{0}=2 \pi c / \lambda_{0}$ the input pulse central frequency and $\phi_{0}$ the carrier-envelope phase.

Figure S6 shows the angularly-resolved temporal intensity profile of the multiple attosecond pulses that are produced in the far field. For the calculation, the laser pulse propagates through a 100- $\mu$ m-thick gas slice with 10-mbar neon pressure. The longitudinal initial atomic density profile is super-Gaussian. The incident laser beam parameters are the following: $\mathrm{R}=$ 25-mm, $\tau=5$-fs (FWHM), $\lambda_{0}=760-\mathrm{nm}, \xi=0.175$-fs $/ \mathrm{mm}$ and $\phi_{0}=\pi / 2$. The peak intensity of the laser pulse is chosen to be $5.0 \times 10^{14} \mathrm{~W} / \mathrm{cm}^{2}$ in order to remain below the saturation intensity of neon gas which is $8.6 \times 10^{14} \mathrm{~W} / \mathrm{cm}^{2}$. The beam is focused $200-\mu \mathrm{m}$ in front of the gas target with a $1-\mathrm{m}$ focal length lens. We used $2^{13}$ points in time, 300 points in the transverse direction and 100 points in the propagation (longitudinal) direction. This simulation shows that pulses of attosecond duration are indeed still generated in the presence of laser wavefront rotation, even when taking into account propagation effects. In addition, these multiple attosecond pulses are well separated angularly, leading to multiple beamlets consisting of isolated attosecond pulses. 


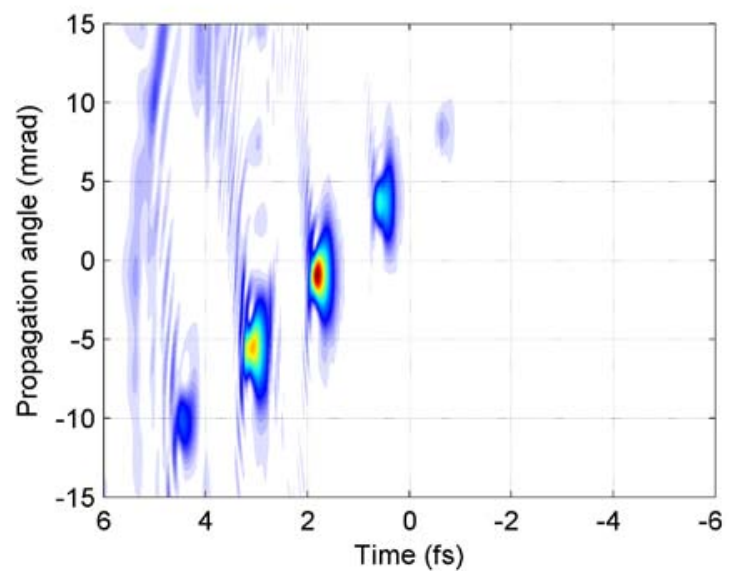

FIG. S6: Angularly resolved temporal intensity profile of the attosecond pulses in the far-field, calculated with the full propagation code, in the presence of WFR.

\section{SUPPLEMENTARY MOVIE}

\section{Angularly-resolved XUV spectrum generated in Ne as a function of the CEP} of the laser pulse. This movie shows the evolution of the multiple angularly-resolved XUV spectra measured from Ne in the experiment as the CEP of the laser pulse is scanned. The CEP is controlled from 0 to $4 \pi$ with 58 steps by adjusting the position of the wedge. Each spectrum is averaged for 30 laser shots. (AVI movie).

[1] V. S. Yakovlev, M. Ivanov, and F. Krausz, Opt. Express 15, 15351 (2007).

[2] G. L. Yudin and M. Y. Ivanov, Phys. Rev. A 64, 013409 (2001).

[3] H. J. Shin, D. G. Lee, Y. H. Cha, K. H. Hong, and C. H. Nam, Phys. Rev. Lett. 83, 2544 (1999).

[4] E. Priori, G. Cerullo, M. Nisoli, S. Stagira, S. De Silvestri, P. Villoresi, L. Poletto, P. Ceccherini, C. Altucci, R. Bruzzese, et al., Physical Review A 61, 063801 (2000).

[5] M. Gaarde, K. Schafer, A. Heinrich, J. Biegert, and U. Keller, Physical Review A 72, 013411 (2005).

[6] M. Lewenstein, P. Balcou, M. Y. Ivanov, A. L'Huillier, and P. B. Corkum, Phys. Rev. A 49, 2117 (1994).

[7] M. V. Ammosov, N. B. Delone, and V. P. Krainov, Zh. Eksp. Teor. Fiz. 91, 2008 (1986).

[8] B. Henke, E. Gullikson, and J. Davis, Atomic data and nuclear data tables 54, 181 (1993). 